ORIGINAL ARTICLE

\section{Early-Onset Stroke and Vasculopathy Associated with Mutations in ADA2}

\author{
Q. Zhou, D. Yang, A.K. Ombrello, Andrey V. Zavialov, C. Toro, Anton V. Zavialov, \\ D.L. Stone, J.J. Chae, S.D. Rosenzweig, K. Bishop, K.S. Barron, H.S. Kuehn, \\ P. Hoffmann, A. Negro, W.L. Tsai, E.W. Cowen, W. Pei, J.D. Milner, C. Silvin, \\ T. Heller, D.T. Chin, N.J. Patronas, J.S. Barber, C.-C.R. Lee, G.M. Wood, A. Ling, \\ S.J. Kelly, D.E. Kleiner, J.C. Mullikin, N.J. Ganson, H.H. Kong, S. Hambleton, \\ F. Candotti, M.M. Quezado, K.R. Calvo, H. Alao, B.K. Barham, A. Jones, \\ J.F. Meschia, B.B. Worrall, S.E. Kasner, S.S. Rich, R. Goldbach-Mansky, M. Abinun, \\ E. Chalom, A.C. Gotte, M. Punaro, V. Pascual, J.W. Verbsky, T.R. Torgerson, \\ N.G. Singer, T.R. Gershon, S. Ozen, O. Karadag, T.A. Fleisher, E.F. Remmers, \\ S.M. Burgess, S.L. Moir, M. Gadina, R. Sood, M.S. Hershfield, M. Boehm, \\ D.L. Kastner, and I. Aksentijevich
}

\section{A B S TRACT}

\section{BACKGROUND}

We observed a syndrome of intermittent fevers, early-onset lacunar strokes and other neurovascular manifestations, livedoid rash, hepatosplenomegaly, and systemic vasculopathy in three unrelated patients. We suspected a genetic cause because the disorder presented in early childhood.

\section{METHODS}

We performed whole-exome sequencing in the initial three patients and their unaffected parents and candidate-gene sequencing in three patients with a similar phenotype, as well as two young siblings with polyarteritis nodosa and one patient with small-vessel vasculitis. Enzyme assays, immunoblotting, immunohistochemical testing, flow cytometry, and cytokine profiling were performed on samples from the patients. To study protein function, we used morpholino-mediated knockdowns in zebrafish and short hairpin RNA knockdowns in U937 cells cultured with human dermal endothelial cells.

RESULTS

All nine patients carried recessively inherited mutations in CECR1 (cat eye syndrome chromosome region, candidate 1), encoding adenosine deaminase 2 (ADA2), that were predicted to be deleterious; these mutations were rare or absent in healthy controls. Six patients were compound heterozygous for eight CECR1 mutations, whereas the three patients with polyarteritis nodosa or small-vessel vasculitis were homozygous for the p.Gly47Arg mutation. Patients had a marked reduction in the levels of ADA2 and ADA2-specific enzyme activity in the blood. Skin, liver, and brain biopsies revealed vasculopathic changes characterized by compromised endothelial integrity, endothelial cellular activation, and inflammation. Knockdown of a zebrafish ADA2 homologue caused intracranial hemorrhages and neutropenia - phenotypes that were prevented by coinjection with nonmutated (but not with mutated) human CECR1. Monocytes from patients induced damage in cocultured endothelial-cell layers.

CONCLUSIONS

Loss-of-function mutations in CECR1 were associated with a spectrum of vascular and inflammatory phenotypes, ranging from early-onset recurrent stroke to systemic vasculopathy or vasculitis. (Funded by the National Institutes of Health Intramural Research Programs and others.)
The authors' full names, degrees, and affiliations are listed in the Appendix. Address reprint requests to $D r$. Aksentijevich at the National Institutes of Health, National Human Genome Research Institute, Bldg. 10, Bethesda, MD 20892, or at askentii@exchange.nih.gov.

Drs. Zhou and Yang and Drs. Boehm, Kastner, and Aksentijevich contributed equally to this article.

This article was published on February 19, 2014, at NEJM.org.

N EnglJ Med 2014;370:911-20. DOI: 10.1056/NEJMoa1307361 Copyright (๑) 2014 Massachusetts Medical Society. 
P ATIENTS WITH AUTOINFLAMMATORY disease sometimes present with clinical findings that encompass multiple organ systems. ${ }^{1}$ Three unrelated children presented to the National Institutes of Health (NIH) Clinical Center with intermittent fevers, recurrent lacunar strokes, elevated levels of acute-phase reactants, livedoid rash, hepatosplenomegaly, and hypogammaglobulinemia. Collectively, these findings do not easily fit with any of the known inherited autoinflammatory diseases.

Hereditary or acquired vascular disorders can have protean manifestations yet be caused by mutations in a single gene. Diseases such as the Aicardi-Goutières syndrome, ${ }^{2,3}$ polypoidal choroidal vasculopathy, ${ }^{4}$ sickle cell anemia, ${ }^{5}$ livedoid vasculopathy, ${ }^{6}$ and the small-vessel vasculitides ${ }^{7,8}$ are examples of systemic diseases that may present with substantial vascular pathologic findings. In some cases, these disorders are not diagnosed until a central nervous system event occurs. Such potentially catastrophic neurologic events often prompt the search for other coincidental findings that may enable a definitive diagnosis. We used whole-exome sequencing to screen for a common genetic cause of disease in these patients. Our investigations led to the discovery of a pathway linking inflammation with vascular development.

\section{METHODS}

\section{PATIENTS}

We evaluated the initial three patients and their unaffected parents, as well as three patients with a similar phenotype, two young siblings with polyarteritis nodosa, and one patient with smallvessel vasculitis. Five of the patients were evaluated at the NIH Clinical Center, and the other four at the Great North Children's Hospital in the United Kingdom and the Hacettepe University Children's Hospital in Turkey. All the patients were enrolled in a study that had been approved by the respective institutional review board. All the patients or their parents provided written informed consent. All the authors vouch for the accuracy and completeness of the data and analyses reported and for the fidelity of the study to the protocol. Lacunar strokes were defined according to standard criteria. ${ }^{9}$

\section{GENETIC AND FUNCTIONAL ANALYSIS}

We performed whole-exome sequencing in the initial three patients and their unaffected parents and candidate-gene sequencing in the other six patients. Enzyme assays, immunoblotting, immunohistochemical testing, flow cytometry, and cytokine profiling were performed on samples from the patients. To study protein function, we used morpholino-mediated knockdowns in zebrafish and short hairpin RNA (shRNA) knockdowns in U937 cells cultured with human dermal endothelial cells. The Supplementary Appendix (available with the full text of this article at NEJM.org) describes the methods used for all these procedures.

\section{RESULTS}

\section{CLINICAL PHENOTYPE}

Initially, three patients with recurrent fevers and early-onset stroke were evaluated; the first patient presented to the NIH at 3 years of age, and the other two at 6 years of age. After they had undergone whole-exome sequencing, two additional patients with a similar phenotype were recruited. All five patients were unrelated, with no history of consanguinity, and represented sporadic cases of European ancestry (i.e., were not in a founder population); all the patients underwent extensive clinical assessment (Tables S1 through S4 in the Supplementary Appendix).

The parents and siblings of the patients from all five families were healthy. All the patients had presented with recurrent fevers and livedo racemosa (Fig. 1A) in early childhood. Skin biopsies revealed a predominance of neutrophils and macrophages in the interstitium, with perivascular $\mathrm{T}$ lymphocytes, without frank vasculitis (Fig. S1A in the Supplementary Appendix). In one patient, necrotizing vasculitis was present in the deep dermis (Fig. 1B and 1C, and Fig. S1B, S1C, and S1D in the Supplementary Appendix). During febrile episodes, the levels of acute-phase reactants were markedly elevated in all the patients.

Each patient had had strokes before 5 years of age (Tables S1 and S3 in the Supplementary Appendix). The strokes occurred mainly during episodes of inflammation, although fever was not always observed. Magnetic resonance imaging (MRI) of the brain showed evidence of acute or chronic small subcortical infarcts ${ }^{10}$ involving the deep-brain nuclei and the brain stem, which are consistent with small-vessel occlusions (lacunar strokes) $^{9,10}$ (Fig. 1D, 1E, and 1F, and Fig. S2A, S2B, and S2C in the Supplementary Appendix). As observed on MRI, the subcortical white matter was spared. Two patients who underwent examination 


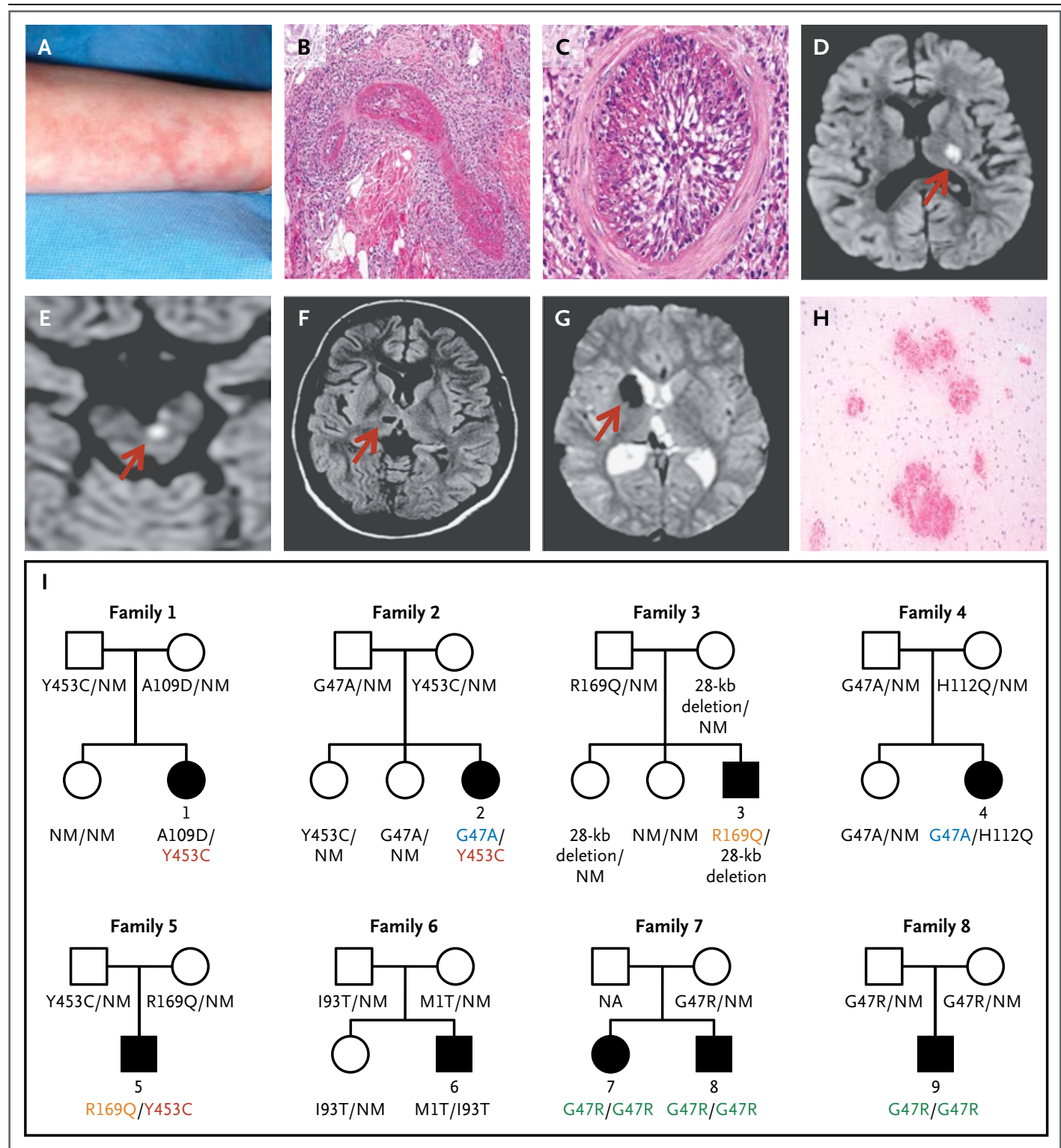

Figure 1. Clinical Findings and Pedigrees of Patients with Deficiency of Adenosine Deaminase 2 (ADA2).

Panel A shows livedo racemosa in Patient 1. Panels B and C show the results of a skin-punch biopsy of the left leg of Patient 4, which revealed vasculitis involving a medium-size blood vessel in the reticular dermis. Panel $B$ shows a dense inflammatory infiltrate with fibrinoid necrosis, and Panel C, the complete occlusion of the vascular lumen (hematoxylin and eosin stain). Panels $D$ through $G$ show the results of brain imaging. Panels $D$ and $E$ show diffusion-weighted axial magnetic resonance images indicating acute small-vessel ischemia in the brain, with Panel D showing ischemia over the left thalamus and posterior limb of the left internal capsule in Patient 5 and Panel $E$ showing ischemia in the left paramedian ventral midbrain in Patient 3; corresponding apparent diffusion coefficient maps are not shown. Panel $F$ shows chronic ischemic changes on axial $\mathrm{T}_{1}$-weighted images in Patient 4 in the form of a small, oval cavitation (lacune) over the right thalamus that resulted from a prior small-vessel occlusion. Panel $\mathrm{G}$ shows hemorrhagic events in Patient 2, as detected on $\mathrm{T}_{2}{ }^{*}$-weighted (gradient echo) images. The localized areas of hypointensity over the right caudate head and posterior thalamus correspond to areas of focal accumulation of hemosiderin due to intraparenchymal bleeding. The arrows in Panels $\mathrm{D}$ through $\mathrm{G}$ point to the area of abnormality. Panel $\mathrm{H}$ shows multiple foci of petechial hemorrhages around small-size vessels in the white matter of the brain in Patient 5 (hematoxylin and eosin stain). Panel I shows the pedigrees of the nine affected patients. Patients 1 through 6 were compound heterozygous for eight mutations in CECR 1 (cat eye syndrome chromosome region, candidate 1), and Patients 7,8 , and 9 were homozygous for one CECR1 mutation. Squares denote male family members, circles female family members, solid symbols affected family members, and open symbols unaffected family members. Shared CECR1 mutations are color-coded. NM denotes nonmutated. 
of the cerebrospinal fluid during acute events had mild lymphocytic pleocytosis.

Several stroke events were hemorrhagic or underwent hemorrhagic transformation, leading to disabling sequelae in some patients (Fig. 1G, and Fig. S2D, S2E, and S2F in the Supplementary Appendix). The interpretation of intracranial bleeding as a phenotypic feature was complicated by the concomitant use of antiplatelet agents, warfarin, or both, although small, deep hemorrhages are increasingly recognized as being within the spectrum of lacunar disease. ${ }^{10,11}$ In all five patients, magnetic resonance angiography showed no evidence of cerebral vasculitis; the absence of cerebral vasculitis was corroborated by conventional angiography in three patients. Computed tomography revealed no calcifications of the basal ganglia. In two patients who underwent biopsy of the brain, there was prominent extravasation of erythrocytes into the Virchow-Robin spaces and white matter around small vessels, without clinically significant inflammation (Fig. 1H). Three patients had ophthalmologic involvement (Table S1 in the Supplementary Appendix).

Four patients presented with hepatosplenomegaly (Fig. S3A, S3B, and S3C in the Supplementary Appendix). One patient had portal hypertension, with hepatofugal flow between the left portal and umbilical veins (Fig. S3D in the Supplementary Appendix). Liver biopsy revealed endothelialization of the hepatic sinusoids (Fig. S3E and S3F in the Supplementary Appendix).

Four patients presented with hypogammaglobulinemia, and two had recurrent bacterial and viral infections before immunosuppressive treatment was initiated. Four patients had varying degrees of lymphopenia. IgM levels were consistently low in all five patients (Table S4 in the Supplementary Appendix).

Hypertension, cardiogenic embolism, and diabetes were ruled out, and extensive hematologic studies did not show hypercoagulability. At the onset of strokes, all the patients were negative for antiphospholipid antibodies (Table S4 in the Supplementary Appendix). Over time, lupus anticoagulant developed in four patients. High doses of glucocorticoids only partially controlled fever, rash, and acute-phase reactants. Despite aggressive treatment with glucocorticoids, cyclophosphamide, and cytokine inhibitors, Patient 4 had her most serious event at 23 years of age.

\section{CECR1 MUTATIONS}

Whole-exome sequencing was performed in Patients 1 and 2 and their unaffected parents (Fig. 1I). After filtering for novel and rare variants (allele frequency, <1\%), we identified approximately 1700 candidate variants in each trio. We hypothesized that the disorder could be caused by either de novo or recessive mutations. A single common candidate gene was identified only under the recessive model (Fig. S4A in the Supplementary Appendix). Both patients were compound heterozygous for missense mutations in CECR1, encoding ADA2 (Fig. S4B in the Supplementary Appendix), and shared the p.Tyr453Cys mutation. All four parents were carriers; the unaffected sibling in Family 1 was a noncarrier, and both unaffected siblings in Family 2 were carriers. We confirmed all variants by means of Sanger sequencing (Fig. S4C in the Supplementary Appendix).

We also performed whole-exome sequencing in Patient 3. The only gene in common with a gene in Patient 1 or Patient 2 that exhibited a deleterious mutation was CECR1. Patient 3 was heterozygous for the p.Arg169Gln variant, which had been inherited from his father. A highdensity single-nucleotide polymorphism array identified a 28-kb deletion in Patient 3 and his mother that included the $5^{\prime}$ untranslated region and exon 1 of CECR1. The deletion breakpoint was confirmed by means of genomic sequencing (Fig. S4C in the Supplementary Appendix). Complementary DNA sequencing showed that the maternal allele carrying the deletion was not expressed. Candidate-gene sequencing in Patients 4 and 5 identified one novel variant, p.His112Gln, and three mutations previously found in Patients 1, 2, and 3 (Fig. 1I). Three of the five missense CECR1 mutations were shared among these five patients.

We subsequently performed candidate-gene sequencing on samples from four additional patients from other centers (Fig. 1I, and Table S5 in the Supplementary Appendix). Patient 6, a 15-year-old boy from the United Kingdom, had recurrent fever, lacunar strokes, and cutaneous manifestations that were different from those observed among the patients at the NIH (Fig. S5A and S5B in the Supplementary Appendix); he died in 2013 from complications of his disease. He was compound heterozygous for the p.Met1Thr null allele and p.Ile93Thr. Patients 7 and 8 were siblings from Turkey with a history of strokes who 
met the criteria for polyarteritis nodosa and were referred on the basis of the work by Navon Elkan and colleagues, now reported in the Journal. ${ }^{12}$ These patients, as well as a Turkish patient with small-vessel vasculitis but no history of stroke (Fig. S5C and S5D in the Supplementary Appendix), were homozygous for the p.Gly47Arg mutation (Fig. 1I, and Fig. S4C in the Supplementary Appendix). Although the p.Gly47Arg variant has not been reported in Europeans, we found that the carrier frequency of this variant in Turkish controls was 0.002 . The key clinical features of all nine patients seen at the NIH and outside centers are summarized in Table 1.

Conserved haplotypes suggested common ancestries for the shared p.Gly47Ala, p.Gly47Arg, p.Arg169Gln, and p.Tyr453Cys mutations (Fig. S6 in the Supplementary Appendix). Three of these mutations were found at low frequencies in the 1000 Genomes Project, the GO Exome Sequencing Project (ESP6500) of the National Heart, Lung, and Blood Institute, and the ClinSeq Project (Table S6 in the Supplementary Appendix). All the variants were computationally predicted to be likely to disrupt function (Table S6 in the Supplementary Appendix), and they affect evolutionarily conserved residues (Fig. S7A in the Supplementary Appendix). We examined individuallevel data from the ESP6500 to explore the CECR1 mutations reported there. In the Siblings with Ischemic Stroke Study (SWISS) ${ }^{13}$ subcohort that included 94 patients with late-onset stroke (Genotypes and Phenotypes database, National Center for Biotechnology Information accession number, phs000327.v1.p1.c1), 2 brothers with ischemic lacunar strokes were heterozygous carriers for the p.Tyr453Cys mutation in ADA2 that was seen in 3 of our pediatric patients (Fig. S8 in the Supplementary Appendix).

\section{FUNCTIONAL STUDIES OF MUTANT ADA2}

ADA2 has partial structural homology with human ADA1. Both proteins convert adenosine to inosine and 2'-deoxyadenosine to 2'-deoxyinosine, but the affinity of ADA2 for adenosine is lower than that of ADA1 by a factor of approximately 100. Whereas ADA1 is monomeric and largely intracellular, ADA2 is dimeric and secreted. Inherited ADA1 deficiency causes profound lymphopenia and severe combined immunodeficiency disease (SCID), which is associated with the toxic intracellular accumulation of deoxyadenosine nucleotides. ${ }^{14}$

\begin{tabular}{|lc|}
\hline $\begin{array}{l}\text { Table } 1 . \text { Clinical and Laboratory Manifestations } \\
\text { in Patients with Deficiency of Adenosine Deaminase } 2 .\end{array}$ & Patients \\
\hline Clinical Manifestation & no.total no. \\
& $9 / 9$ \\
Fever & $8 / 9$ \\
Ischemic stroke & $3 / 9$ \\
Hemorrhagic stroke & $5 / 9$ \\
Ophthalmologic involvement* & $8 / 9$ \\
Livedo racemosa & $6 / 7$ \\
Hepatosplenomegaly & $4 / 9$ \\
Documented vasculitis $\dagger$ & $2 / 9$ \\
Polyarteritis nodosa & $3 / 9$ \\
Antinuclear antibody & $0 / 9$ \\
Antineutrophil cytoplasmic antibody & $5 / 5$ \\
Low serum IgM & \\
\hline
\end{tabular}

* The ophthalmologic manifestations included central retinal artery occlusion in one patient, optic nerve atrophy in one, diplopia with irregular enhancement of the medial rectus muscle (as observed on magnetic resonance imaging) in one, third cranial nerve palsy in one, and strabismus in two. Patients could have more than one ophthalmologic disorder.

$\uparrow$ The diagnosis of vasculitis included polyarteritis nodosa.

Computer modeling of the missense mutations presented here, which was based on the crystal structure of human ADA2, ${ }^{15}$ suggests that these mutations are probably loss-of-function mutations (Fig. S7B and S7C in the Supplementary Appendix), affecting the catalytic and dimerization domains and protein stability. Using two independent assays, we measured ADA2 activity in plasma and serum samples from the patients at the NIH, their relatives, and healthy adult and pediatric controls. The patients had significantly diminished ADA2 activity in plasma (Fig. 2A). Western blot analysis showed that the ADA2 protein was reduced in cell lysates and absent from the supernatants of macrophages from patients from whom cultures were obtained (Fig. S9 in the Supplementary Appendix).

In assays measuring ADA activity in monocytes and plasma from the patients, a significant reduction was observed only in ADA2-specific activity, whereas ADA1-specific activity was preserved (Fig. S10A and S10B and Table S7 in the Supplementary Appendix). The results of thinlayer chromatographic assays for ADA1 activity in erythrocyte hemolysates were normal, and the accumulation of deoxyadenosine nucleotides, which is the biochemical hallmark of ADA1 de- 


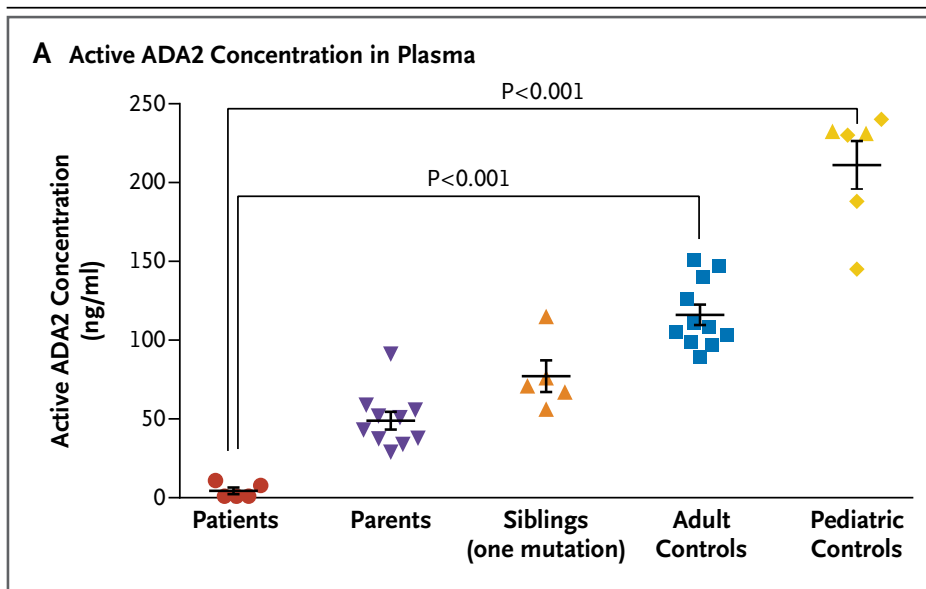

B Confocal Microscopy in Zebrafish Uninjected
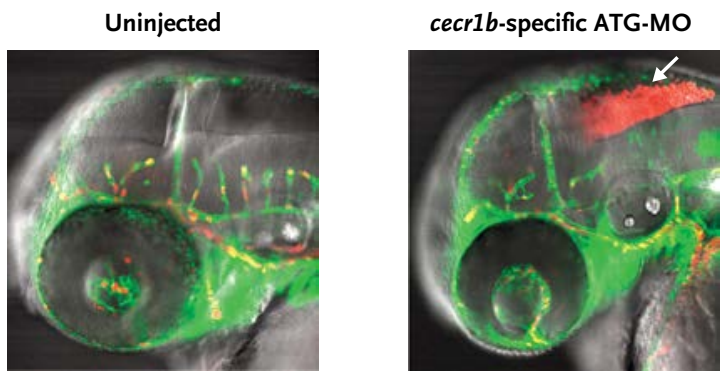

Figure 2. Evidence of Loss-of-Function Mutations in ADA2.

Panel A shows ADA2 activity in plasma samples from five patients with stroke (red circles), relatives who are clinically asymptomatic carriers of one mutation (purple triangles and orange triangles), healthy adult controls (blue squares), and healthy pediatric controls (yellow diamonds) (see the Supplementary Appendix). Two siblings (yellow triangles) who are not carriers of ADA2 mutations clustered appropriately with pediatric controls. Plasma specimens were titrated against recombinant ADA2 standards to quantitate the amount of nonmutant protein that would give equivalent activity. The horizontal lines represent mean values, and the I bars the standard error. Panel B shows confocal microscopy in zebrafish. Transgenic expression of enhanced green fluorescent protein under the fli1 promoter labels blood vessels green; expression of red fluorescent protein from discosoma species under the gata1 promoter labels erythrocytes red. Intracranial bleeding (arrow) is observed in embryos injected with cecrlb-specific morpholino oligonucleotides, targeting the translation initiation site (ATG-MO). Similar results were observed for embryos injected with the splice-blocking morpholinos targeting the proper splicing of exon 3.

ficiency, was absent in erythrocytes from patients with ADA2 deficiency (Fig. S10C and S10D in the Supplementary Appendix).

IMMUNOLOGIC ASSESSMENT OF ADA2 DEFICIENCY

Extensive studies of serum cytokines or of cytokines produced by cultured peripheral-blood mononuclear cells did not show any convincing differences between findings in the patients and those in healthy controls (Fig. S11 in the Sup- plementary Appendix). Given the profound T-cell defects in ADA1 deficiency, ${ }^{16}$ we examined thymic output and T-cell function. Recent thymic emigrants (CD3+, CD31+, and CD45RA+) and naive $\mathrm{T}$ cells $(\mathrm{CD} 3+, \mathrm{CD} 62 \mathrm{~L}+$, and $\mathrm{CD} 45 \mathrm{RA}+)$ were normal (data not shown). Patients with ADA2 deficiency had normal short-term T-cell activation and normal proliferative responses to antiCD3 antibodies (Fig. S12 in the Supplementary Appendix). They had slightly increased proliferation in both CD4+ and CD8+ subsets in response to phytohemagglutinin stimulation but normal production of effector cytokines.

When peripheral-blood mononuclear cells were cultured for 48 hours without stimulation, higher rates of spontaneous B-cell death were seen in the samples from the patients than in the samples from healthy controls (Fig. S13 in the Supplementary Appendix). As compared with samples from age-matched controls, samples from patients had fewer memory B cells in the peripheral blood, lower expression of CD27 and IgG on B cells after induction with a variety of stimuli, and a modest reduction in the terminal differentiation of B cells and immunoglobulin-secreting cells after T-cell-dependent stimulation (Fig. S14 in the Supplementary Appendix). A bone marrow aspirate and biopsy performed in one patient revealed normal B-cell maturation but reduced numbers of CD138+ plasma cells (data not shown).

\section{AN ANIMAL MODEL OF ADA2 DEFICIENCY}

Although there is no murine orthologue of CECR1, there are two paralogs in zebrafish: cecrla (chromosome 25) and cecr1b (chromosome 4). A cecr1a hypomorphic line generated by means of random insertional mutagenesis with a retroviral vector (Fig. S15A, S15B, and S15C in the Supplementary Appendix) had no overt phenotype. We developed a transient knockdown strategy for cecr1b using translation-blocking and splice-blocking morpholinos (Fig. S16A in the Supplementary Appendix). A reverse-transcriptase-polymerase-chain-reaction assay of embryos injected with morpholino antisense strategies to prevent the proper splicing of exon 3 (E3I3-MO) confirmed the ability of the antisense construct to induce skipping of exon 3 in the cecr1b transcript (Fig. S16B in the Supplementary Appendix), resulting in a frameshift, premature termination, and a reduced transcript level of $8 \%$ of nonmutant expression (Fig. S15C in the Supplementary Appendix).

We observed intracranial bleeding (Fig. 2B) 
with both cecr1b morpholinos in a dose-dependent manner (Fig. S16C in the Supplementary Appendix), which suggested the presence of defects in vessel development or integrity. ${ }^{17}$ Blood-vessel morphologic features appeared to be normal, despite evidence of hemorrhage and ischemia in embryos injected with cecr $1 b$-specific morpholino oligonucleotides targeting the translation initiation site (ATG-MO) (Fig. S16D in the Supplementary Appendix). Intracranial hemorrhage was blocked by coinjection with nonmutant human CECR1 messenger RNA (mRNA) but not with mutant transcripts (Fig. S16E in the Supplementary Appendix).

Zebrafish that were severe hypomorphs for cecr1a showed no significant increase in intracranial bleeding; in addition, when embryos that were hypomorphs for cecr1a were injected with cecr1b morpholinos, there was no significant increase in intracranial bleeding in these embryos as compared with their morpholino-injected nonmutant siblings (data not shown), suggesting that cecr $1 b$ primarily provides the ADA2 function necessary to prevent the hemorrhaging phenotypes. The two paralogs showed different expression patterns, suggesting that they have functionally diverged (Fig. S15D and S15E in the Supplementary Appendix).

When ATG-MO was used to disrupt cecr1b expression in mpx:EGFP transgenic zebrafish (with the gene for enhanced green fluorescent protein under the control of a myeloid-specific peroxidase promoter), in which neutrophils are labeled green, there was marked neutropenia (Fig. S16F in the Supplementary Appendix). This phenotype was blocked by the coinjection of nonmutant human CECR1 mRNA but not by the coinjection of H112Q or R169Q mRNA, both of which are associated with stroke in our patients.

\section{EFFECTS OF ADA2 DEFICIENCY ON ENDOTHELIAL AND LEUKOCYTE DEVELOPMENT}

Given the zebrafish data, we examined endothelial integrity and activation in patients. Staining with anti-CD31 antibodies showed substantial endothelial damage in biopsy specimens from the brain (Fig. S17A, S17D, S17J, and S17M in the Supplementary Appendix) and from lesional skin (Fig. S17B, S17C, S17E, S17F, S17K, S17L, S17N, and S17O in the Supplementary Appendix). Endothelial-cell activation as shown by E-selectin staining was observed both in brain-biopsy specimens (Fig. 3A, and Fig. S17G in the Supplementary Appendix) and in skin-biopsy specimens
(Fig. 3B and 3C, and Fig. S17H and S17I in the Supplementary Appendix). There was also increased staining for interleukin-1 $\beta$ (Fig. 3D, 3E, and $3 \mathrm{~F}$ ), inducible nitric oxide synthase (Fig. S18A, S18B, and S18C in the Supplementary Appendix), and tumor necrosis factor $\alpha$ (Fig. S18D, S18E, and S18F in the Supplementary Appendix), indicating inflammation.

CECR1 is not expressed, nor is the ADA2 protein detectable, in cultured human endothelial cells (Fig. S19 in the Supplementary Appendix). This implicates additional cell types in the vasculopathy. Previous studies have shown a role for human ADA2 in the differentiation of monocytes to macrophages. ${ }^{18}$ Using shRNA constructs to silence the expression of ADA2 in myeloid U937 cells, we observed marked impairment of macrophage differentiation induced by phorbol myristate acetate (Fig. S20A, S20B, and S20C in the Supplementary Appendix). Under standard conditions, ${ }^{19}$ monocytes from the patients differentiated into M1 macrophages but poorly differentiated into M2 macrophages (Fig. 3G through J, and Fig. S21 in the Supplementary Appendix), which may have proinflammatory effects. ${ }^{20,21}$ Furthermore, shRNA knockdown of ADA2 in U937 cells led to considerable disruption of cocultured monolayers of human primary dermal microvascular endothelial cells (Fig. S22A in the Supplementary Appendix), as did monocytes from two patients with ADA2 deficiency (Fig. 3K and 3L, and Fig. S22B in the Supplementary Appendix).

\section{DISCUSSION}

We describe a disorder characterized by recurrent fevers, a spectrum of vascular pathologic features, and mild immunodeficiency. The data implicate loss-of-function mutations in CECR1, encoding the ADA2 protein (Table S8 in the Supplementary Appendix). Studies in patients' cells and in zebrafish support the hypothesis that ADA2 is a growth factor for endothelial and leukocyte development and differentiation. As compared with patients with ADA1 deficiency, those with ADA2 deficiency have only a mild immunodeficiency that is most evident in B cells.

Our data corroborate a role for ADA2 as the prototype for a family of adenosine deaminaserelated growth factors, a role that had previously been suggested by studies in xenopus and drosophila. ${ }^{22-24}$ The experiments described here indicate that cecr1b is essential for both vascular 


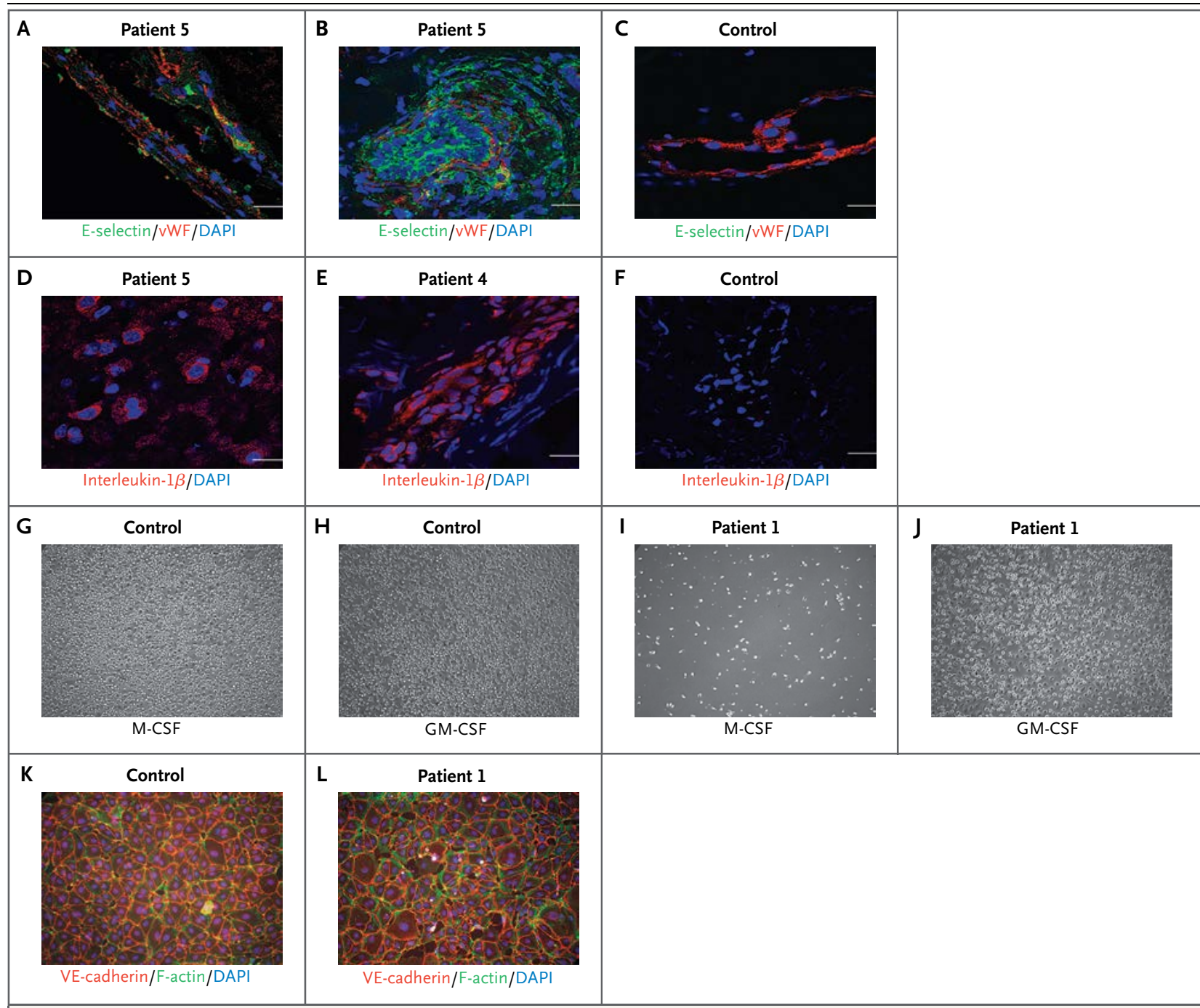

Figure 3. Effect of ADA2 Deficiency in Patients.

Cells stained with endothelial-cell activation marker E-selectin (green) are shown in a brain-biopsy sample from Patient 5 (Panel A) and in skin-biopsy samples from Patient 5 (Panel B) and a control donor (Panel C). E-selectin in endothelial cells (expressing von Willebrand factor [VWF; red]) indicates endothelial-cell activation (Panels A and B). E-selectin is absent in endothelial cells from a healthy control (Panel C). Nuclei are stained blue with 4',6-diamidino-2-phenylindole (DAPI). Scale bars indicate $20 \mu \mathrm{m}$. Interleukin-1 $\beta$ immunostaining (red) is shown in a brain-biopsy sample from Patient 5 (Panel D) and in skin-biopsy samples from Patient 4 (Panel E) and a control donor (Panel F). Positive cells can be seen in the brain sample from Patient 5 (Panel D), and robust interleukin- $\beta$ staining is seen in a skinbiopsy sample from Patient 4 (Panel E). An Ml and M2 differentiation assay (Panels $\mathrm{G}$ through J) was performed in monocytes that had been isolated by means of negative selection from blood samples from Patient 1 and an age-matched control. Equal cell numbers were seeded; M2 macrophage differentiation was induced with $50 \mathrm{ng}$ per milliliter of macrophage colony-stimulating factor (M-CSF), and M1 macrophage differentiation with $20 \mathrm{ng}$ per milliliter of granulocyte-macrophage colony-stimulating factor (GM-CSF) for 10 days. Control monocytes attached and differentiated, showing macrophage-like morphologic features under both M-CSF and GM-CSF stimulation (Panels G and H). Very few attached and differentiated M2-like cells were observed in M-CSF-stimulated monocytes from Patient 1 (Panel I). However, Ml-like cells were observed in GM-CSF-induced differentiation of monocytes from Patient 1 (Panel J) — similar to those seen in control cells. Human dermal microvascular endothelial cells were grown to confluence and cocultured with monocytes isolated from a control donor and from Patient 1 for 3 days. Nonadherent cells were removed, and the endothelial-cell layers were stained for the endothelial junction protein VE-cadherin (red), F-actin (green), and DAPI (blue). Endothelial cells cultured with healthy control monocytes (Panel K) show normal cell layers, whereas endothelial cells cocultured with monocytes isolated from Patient 1 show damaged, interrupted endothelial cell layers (Panel L). 
integrity and neutrophil development in the zebrafish embryo and that both phenotypes are prevented by nonmutant, but not by mutant, human CECR1 mRNA.

Moreover, although ADA2 is not expressed in endothelial cells, there is a defect in endothelial integrity in the small vessels of patients with ADA2 mutations as well as impairment of M2 macrophage differentiation. ADA2 is known to be produced by myeloid cells and to promote macrophage differentiation. ${ }^{18}$ Together, the data from zebrafish and patients suggest that ADA2 deficiency may compromise endothelial integrity while polarizing macrophage and monocyte subsets toward proinflammatory cells, establishing a vicious circle of vasculopathy and inflammation..$^{20,21,25}$

Given the spectrum of disease observed in these nine patients, $\mathrm{ADA} 2$ may play a role in other disorders. The observation of two brothers who were heterozygous for the p.Tyr453Cys mutation and who had late-onset lacunar strokes warrants larger-scale sequencing studies to explore the significance of CECR1 variants in complex forms of lacunar stroke and vasculitis. It is also possible that ADA2 deficiency accounts for some patients with Sneddon's syndrome, a poorly understood disorder that is most common in middle-age women and that is characterized by livedoid rash and stroke, with antiphospholipid antibodies present in some of the patients. ${ }^{26}$

Therapeutic strategies for the treatment of patients with ADA2 deficiency require investigation. Since the phenotype appears not to be caused by the accumulation of adenosine and deoxyadenosine, treatment with pegylated ADA1, an effective therapy for ADA-related SCID, is unlikely to be successful. However, since ADA2 is found in plasma, these patients may benefit from fresh-frozen plasma or recombinant ADA2, assuming that cell-cell interactions are not necessary for effective ADA2 activity. Alternatively, given that monocytes and macrophages, the main producers of ADA2, are derived from bone marrow, it is possible that bone marrow transplantation or genetic manipulation of bone marrow cells has a role in the treatment of these patients.

In conclusion, we defined a genetic disorder, for which we propose the name "deficiency of ADA2" (DADA2), that connects systemic inflammation, vascular pathology, and mild immunodeficiency. DADA2 establishes a role for adenosine deaminaserelated growth factors in human disease and provides potential diagnostic and therapeutic strategies for a newly recognized group of patients.

Supported by the National Institutes of Health (NIH) Intramural Research Programs, including the Intramural Research Programs of the National Human Genome Research Institute, the National Institute of Arthritis and Musculoskeletal and Skin Diseases, the National Heart, Lung, and Blood Institute (NHLBI), the National Institute of Allergy and Infectious Diseases, the National Institute of Diabetes and Digestive and Kidney Diseases, the National Cancer Institute, the Undiagnosed Diseases Program of the Common Fund of the Office of the Director of the $\mathrm{NIH}$, and the NIH Clinical Center; and by grants from Sigma Tau Pharmaceuticals (to Dr. Hershfield) and the Finnish Academy (to Drs. Andrey and Anton Zavialov).

Disclosure forms provided by the authors are available with the full text of this article at NEJM.org.

We thank the staff at the NIH Intramural Sequencing Center for performing whole-exome sequencing, Dr. Leslie Biesecker for sharing variant data from the ClinSeq Project, Mr. Stephen Wincovitch for performing confocal microscopy of zebrafish, Ms. Sunny Huang for embryo injections, Dr. Eyal Muscal for clinical support and expertise, Drs. Robert I. Richards and Brant $M$. Weinstein for a critical reading of an earlier version of the manuscript and for helpful discussion, Dr. Christian A. Combs and Dr. Daniela Malide (Light Microscopy Core Facility, NHLBI, $\mathrm{NIH}$ ) for professional skills and advice regarding microscopyrelated experiments, Dr. Zu-Xi Yu and colleagues at the NHLBI Pathology Core Facility for professional skills and advice, and all the affected children and their families, and the healthy children and adult controls, for participation in this research study.

\section{APPENDIX}

The authors' full names and degrees are as follows: Qing Zhou, Ph.D., Dan Yang, M.D., Ph.D., Amanda K. Ombrello, M.D., Andrey V. Zavialov, Ph.D., Camilo Toro, M.D., Anton V. Zavialov, Ph.D., Deborah L. Stone, M.D., Jae Jin Chae, Ph.D., Sergio D. Rosenzweig, M.D., Ph.D., Kevin Bishop, B.Sc., Karyl S. Barron, M.D., Hye Sun Kuehn, Ph.D., Patrycja Hoffmann, N.P., Alejandra Negro, Ph.D., Wanxia L. Tsai, M.S., Edward W. Cowen, M.D., Wuhong Pei, Ph.D., Joshua D. Milner, M.D., Christopher Silvin, Ph.D., Theo Heller, M.D., David T. Chin, B.Sc., Nicholas J. Patronas, M.D., John S. Barber, B.A., Chyi-Chia R. Lee, M.D., Ph.D., Geryl M. Wood, M.A., Alexander Ling, M.D., Susan J. Kelly, Ph.D., David E. Kleiner, M.D., Ph.D., James C. Mullikin, Ph.D., Nancy J. Ganson, Ph.D., Heidi H. Kong, M.D., Sophie Hambleton, Ph.D., Fabio Candotti, M.D., Martha M. Quezado, M.D., Katherine R. Calvo, M.D., Ph.D., Hawwa Alao, M.D., Beverly K. Barham, R.N., M.S.N., Anne Jones, R.N., B.S.N., James F. Meschia, M.D., Bradford B. Worrall, M.D., Scott E. Kasner, M.D., Stephen S. Rich, Ph.D., Raphaela Goldbach-Mansky, M.D., Mario Abinun, M.D., Elizabeth Chalom, M.D., Alisa C. Gotte, M.D., Marilynn Punaro, M.D., Virginia Pascual, M.D., James W. Verbsky, M.D., Ph.D., Troy R. Torgerson, M.D., Ph.D., Nora G. Singer, M.D., Timothy R. Gershon, M.D., Ph.D., Seza Ozen, M.D., Omer Karadag, M.D., Thomas A. Fleisher, M.D., Elaine F. Remmers, Ph.D., Shawn M. Burgess, Ph.D., Susan L. Moir, Ph.D., Massimo Gadina, Ph.D., Raman Sood, Ph.D., Michael S. Hershfield, M.D., Manfred Boehm, M.D., Daniel L. Kastner, M.D., Ph.D., and Ivona Aksentijevich, M.D. 
The authors' affiliations are as follows: National Human Genome Research Institute (Q.Z., A.K.O., C.T., D.L.S., J.J.C., K.B., P.H., W.P., C.S., D.T.C., G.M.W., J.C.M., F.C., B.K.B., A.J., E.F.R., S.M.B., R.S., D.L.K., I.A.), National Heart, Lung, and Blood Institute (D.Y., A.N., M.B.), National Institute of Allergy and Infectious Diseases (S.D.R., K.S.B., J.D.M., J.S.B., S.L.M.), National Institutes of Health Clinical Center (H.S.K., N.J.P., A.L., K.R.C., T.A.F.), National Institute of Arthritis and Musculoskeletal and Skin Diseases (W.L.T., R.G.-M., M.G.), National Cancer Institute (E.W.C., C.-C.R.L., D.E.K., H.H.K., M.M.Q.), and National Institute of Diabetes and Digestive and Kidney Diseases (T.H., H.A.) - all in Bethesda, MD; University of Turku, Turku, Finland (Andrey V. Zavialov, Anton V. Zavialov); Duke University Medical Center, Durham, N.C. (S.J.K., N.J.G., M.S.H.); University of North Carolina Neuroscience Center, Chapel Hill (T.R.G.); Institute of Cellular Medicine, Newcastle University, Newcastle, United Kingdom (S.H., M.A.); Mayo Clinic Florida, Jacksonville (J.F.M.); University of Virginia, Charlottesville (B.B.W., S.S.R.); University of Pennsylvania, Philadelphia (S.E.K.); Barnabas Health, West Orange, NJ (E.C.); Texas Scottish Rite Hospital (A.C.G., M.P.) and Baylor Institute for Immunology Research (V.P.) - both in Dallas; Medical College of Wisconsin, Milwaukee (J.W.V.); University of Washington and Seattle Children's Research Institute, Seattle (T.R.T.); Metro Health Medical Center and Case Western Reserve University School of Medicine, Cleveland (N.G.S.); and Hacettepe University Faculty of Medicine, Ankara, Turkey (S.O., O.K.).

REFERENCES

1. Kastner DL, Aksentijevich I, GoldbachMansky R. Autoinflammatory disease reloaded: a clinical perspective. Cell 2010; 140:784-90.

2. Crow YJ, Rehwinkel J. Aicardi-Goutieres syndrome and related phenotypes: linking nucleic acid metabolism with autoimmunity. Hum Mol Genet 2009;18(R2): R130-R136.

3. du Moulin M, Nürnberg P, Crow YJ, Rutsch F. Cerebral vasculopathy is a common feature in Aicardi-Goutieres syndrome associated with SAMHD1 mutations. Proc Natl Acad Sci U S A 2011;108(26):E232E233.

4. Lima LH, Schubert C, Ferrara DC, et al. Three major loci involved in age-related macular degeneration are also associated with polypoidal choroidal vasculopathy. Ophthalmology 2010;117:1567-70.

5. Kato GJ, Hebbel RP, Steinberg $M H$, Gladwin MT. Vasculopathy in sickle cell disease: biology, pathophysiology, genetics, translational medicine, and new research directions. Am J Hematol 2009; 84:618-25.

6. Khenifer S, Thomas L, Balme B, Dalle S. Livedoid vasculopathy: thrombotic or inflammatory disease? Clin Exp Dermatol 2010;35:693-8.

7. Talarico R, Baldini C, Della Rossa A, et al. Large- and small-vessel vasculitis: a critical digest of the 2010-2011 literature. Clin Exp Rheumatol 2012;30:Suppl 70:S130-S138

8. Federico A, Di Donato I, Bianchi S, Di Palma C, Taglia I, Dotti MT. Hereditary cerebral small vessel diseases: a review. J Neurol Sci 2012;322:25-30.

9. Adams HP Jr, Bendixen BH, Kappelle LJ, et al. Classification of subtype of acute ischemic stroke - definitions for use in a multicenter clinical trial: TOAST: Trial of Org 10172 in Acute Stroke Treatment Stroke 1993;24:35-41.

10. Wardlaw JM, Smith C, Dichgans M. Mechanisms of sporadic cerebral small vessel disease: insights from neuroimaging Lancet Neurol 2013;12:483-97. [Erratum, Lancet Neurol 2013;12:532.]

11. Rost NS, Rahman RM, Biffi A, et al. White matter hyperintensity volume is in creased in small vessel stroke subtypes. Neurology 2010;75:1670-7.

12. Navon Elkan P, Pierce SB, Segel R, et al. Mutant adenosine deaminase 2 in a polyarteritis nodosa vasculopathy. $\mathrm{N}$ Engl Med 2014;370:921-31.

13. Meschia JF, Brown RD Jr, Brott TG Chukwudelunzu FE, Hardy J, Rich SS. The Siblings with Ischemic Stroke Study (SWISS) protocol. BMC Med Genet 2002; 3:1-12.

14. Hershfield M. Combined immune deficiencies due to purine enzyme defects In: Stiehm ER, Ochs HD, Winkelstein JA, eds. Immunologic disorders in infants and children. 5th ed. Philadelphia: W.B. Saunders, 2004:480-504

15. Zavialov AV, Yu X, Spillmann D, Lauvau G, Zavialov AV. Structural basis for the growth factor activity of human adenosine deaminase ADA2. J Biol Chem 2010 285:12367-77.

16. Cassani B, Mirolo M, Cattaneo F, et al. Altered intracellular and extracellula signaling leads to impaired T-cell functions in ADA-SCID patients. Blood 2008, 111:4209-19.

17. Butler MG, Gore AV, Weinstein BM Zebrafish as a model for hemorrhagic stroke. Methods Cell Biol 2011;105:137-61. 18. Zavialov AV, Gracia E, Glaichenhaus N, Franco R, Zavialov AV, Lauvau G. Hu- man adenosine deaminase 2 induces differentiation of monocytes into macrophages and stimulates proliferation of $\mathrm{T}$ helper cells and macrophages. J Leukoc Biol 2010;88:279-90.

19. Rey-Giraud F, Hafner M, Ries CH. In vitro generation of monocyte-derived macrophages under serum-free conditions improves their tumor promoting functions. PLoS One 2012;7(8):e42656.

20. Moore KJ, Tabas I. Macrophages in the pathogenesis of atherosclerosis. Cell 2011;145:341-55.

21. Woollard KJ, Geissmann F. Monocytes in atherosclerosis: subsets and functions. Nat Rev Cardiol 2010;7:77-86.

22. Iijima R, Kunieda T, Yamaguchi S, et al. The extracellular adenosine deaminase growth factor, ADGF/CECR1, plays a role in xenopus embryogenesis via the adenosine/P1 receptor. J Biol Chem 2008;283: 2255-64.

23. Maier SA, Podemski L, Graham SW, McDermid HE, Locke J. Characterization of the adenosine deaminase-related growth factor (ADGF) gene family in drosophila. Gene 2001;280:27-36.

24. Maier SA, Podemski L, Graham SW, McDermid HE, Locke J. Characterization of the adenosine deaminase-related growth factor (ADGF) gene family in Drosophila. Gene 2001;280:27-36.

25. Eltzschig HK, Sitkovsky MV, Robson SC. Purinergic signaling during inflammation. N Engl J Med 2013;368:1260.

26. Francès $\mathrm{C}$, Papo $\mathrm{T}$, Wechsler $\mathrm{B}$, Laporte JL, Biousse V, Piette JC. Sneddon syndrome with or without antiphospholipid antibodies: a comparative study in 46 patients. Medicine (Baltimore) 1999; 78:209-19.

Copyright (c) 2014 Massachusetts Medical Society.

RECEIVE THE JOURNAL'S TABLE OF CONTENTS EACH WEEK BY E-MAIL

To receive the table of contents of the Journal by e-mail every Wednesday evening, sign up at NEJM.org. 